\title{
The impact of uncertainty on optimal emission policies
}

\author{
Nicola Botta ${ }^{1}$, Patrik Jansson ${ }^{2}$, and Cezar Ionescu ${ }^{3}$ \\ ${ }^{1}$ Potsdam Institute for Climate Impact Research, Telegraphenberg A31, 14473 Potsdam, Germany \\ ${ }^{2}$ Chalmers University of Technology, Computer Science and Engineering, 41296 Göteborg, Sweden \\ ${ }^{3}$ University of Oxford, Dept. for Continuing Education, Ewert House, Ewert Place, OX2 7DD, UK
}

Correspondence: Nicola Botta (botta@pik-potsdam.de)

Received: 28 September 2017 - Discussion started: 5 October 2017

Revised: 15 February 2018 - Accepted: 17 March 2018 - Published: 18 May 2018

\begin{abstract}
We apply a computational framework for specifying and solving sequential decision problems to study the impact of three kinds of uncertainties on optimal emission policies in a stylized sequential emission problem. We find that uncertainties about the implementability of decisions on emission reductions (or increases) have a greater impact on optimal policies than uncertainties about the availability of effective emission reduction technologies and uncertainties about the implications of trespassing critical cumulated emission thresholds. The results show that uncertainties about the implementability of decisions on emission reductions (or increases) call for more precautionary policies. In other words, delaying emission reductions to the point in time when effective technologies will become available is suboptimal when these uncertainties are accounted for rigorously. By contrast, uncertainties about the implications of exceeding critical cumulated emission thresholds tend to make early emission reductions less rewarding.
\end{abstract}

\section{Introduction}

\subsection{About this work}

In this article we apply the framework for specifying and solving sequential decision problems (SDPs) presented in Botta et al. (2017b) to understand the impact of uncertainty on optimal greenhouse gas (GHG) emission policies.

Specifically, we study the impact of

1. uncertainty about the implementability of decisions on GHG emission reductions,

2. uncertainty about the availability of efficient technologies for reducing GHG emissions and

3. uncertainty about the implications of exceeding a critical threshold of cumulated GHG emissions.

The work is also an application of the computational theory of policy advice and avoidability proposed in Botta et al. (2017a). The theory supports a seamless approach towards accounting for different kinds of uncertainties and makes it possible to rigorously assess the logical consequences, including the risks, entailed by the implementation of optimal policies. We explain what policies are and what it means for a policy sequence to be optimal in Sect. 2.3.

\subsection{Sequential decision problems and climate change}

In many decision problems in the context of climate change, decisions have to be taken sequentially: emission rights are issued year after year, emission reduction plans and measures are iteratively revised and updated at certain (perhaps irregular) points in time, etc.

In its Fourth Assessment Report IPCC (2007), the Intergovernmental Panel on Climate Change (IPCC) pointed out that responding to climate change involves "an iterative risk management process that includes both mitigation and adaptation, taking into account actual and avoided climate change damages, co-benefits, sustainability, equity and attitudes to risk."

The paradigmatic example of iterative SDPs in the context of climate change is that of controlling GHG emissions. In GHG emission control problems, a decision maker or a finite number of decision makers (countries) have to select an emission level or, equivalently, a level of emission abatement (reduction) with respect to some reference emissions. 
The idea is that the selected abatement level is then implemented, perhaps with some deviations, over a certain period of time. After that period another decision is taken for the next time period.

Implementing abatements implies both costs and benefits. These are typically affected by different kinds of uncertainties but the idea is that, for a specific decision maker, a significant part of the benefits comes from avoided damages from climate change. Avoided damages essentially depend on the overall abatements: higher global abatements lead to less damages and thus higher benefits. In contrast, costs are very much dependent on the abatement level implemented by the specific decision maker. Here, higher emission reductions cost more than moderate emission reductions.

It turns out that, when considering a single decision step and for fairly general and realistic assumptions on how costs and benefits depend on abatement levels, the highest global benefits are obtained if all decision makers reduce emissions by certain "optimal" amounts (Finus et al., 2003; Helm, 2003; Heitzig et al., 2011).

In this situation, however, many (if not all) decision makers typically face a free-ride option: they could do even better if they themselves did not implement any emission reduction (or, perhaps, if they would implement less reductions) but all the others still complied with their quotas. It goes without saying that if all players fail to comply with their optimal emission reduction quotas, the overall outcome will be unsatisfactory for all or most players.

This situation is often referred to as an instance of the "Tragedy of the Commons" Hardin (1968) and has motivated a large body of research on, among other things, coalition formation and the design of mechanisms to deter free-riding. These studies are naturally informed by game-theoretical approaches and focus on the nonparametric nature of decision making. The sequentiality of the underlying decision process and the temporal dimension of decision making are traded for analytic tractability. For a survey, see Heitzig et al. (2011).

Another avenue of research focuses on the investigation of optimal global emission paths or, as we shall see in Sect. 2.3, of optimal sequences of global emission policies. Here, the core question is how uncertain future developments, typically, the introduction of new technologies or the crossing of climate stability thresholds, shall inform current decisions. In a nutshell, the problems here are when global emissions should be reduced and by how much given the uncertainties that affect both our understanding of the earth system and the socioeconomic consequences of implementing emission reductions.

In these kinds of studies, the presence of multiple decision makers with possibly conflicting interests and the question of how emission reductions can actually be implemented is neglected. This makes it possible to apply control theoretical approaches and to fully account for the temporal dimension of sequential emission games. This is also the approach followed in this work. To the best of our knowledge, no theory is currently available for tackling the problem of computing optimal emission policies for individual countries as a (mixed sequential and simultaneous) coordination game with a finite number of decision makers, over a finite (but not necessarily known) number of decision steps and under different sources of uncertainty. For a survey of SDPs under uncertainty in climate change see Parson and Karwat (2011), Peterson (2006) and references therein.

\subsection{Stylized sequential emission problems}

One can try to understand the impact of uncertainties on optimal emission policies for a specific, real (or, more likely, realistic) emission problem. This requires, among other things, specifying an integrated climate-economy assessment model or, as done in Webster (2008), some tabulated version of the model underlying the problem. The approach supports drawing conclusions that are specific for the problem under investigation and is what is typically done in applied policy advice. On the other hand, studying a specific, realistic problem makes it difficult to draw general conclusions and is well beyond the scope of this work.

An alternative approach towards understanding the impacts of uncertainties on optimal policies is to study a "stylized" emission problem. A stylized emission problem does not attempt to be realistic. Instead, it tries to capture the essential features of a whole class of problems and supports general instead of specific conclusions. This is the approach followed in this paper.

\subsection{Notation}

In Sect. 5 we apply the theory for specifying and solving SDPs from Botta et al. $(2017 \mathrm{~b}$, a) to the stylized emission problem from Sect. 4. The theory is based on the notion of monadic dynamical systems originally introduced in Ionescu (2009). In this context, monads allow one to treat deterministic, nondeterministic, stochastic, fuzzy, etc. uncertainty with a seamless approach: the differences are captured by a single problem parameter and all computations are generic with respect to this parameter. In a nutshell, the theory is a dependently typed formalization of dynamic programming (Bellman, 1957). The formalization language is Idris; see Brady (2013). For a discussion on why functional, dependently typed languages are the first choice for implementing such formalizations, see Botta et al. (2017a).

Because the theory is dependently typed, some familiarity with a functional, dependently typed notation is mandatory to apply it to a specific decision problem. In this paper, we do not assume that our readership is familiar with dependent types and functional languages. Thus, in Sects. 2 to 6 , we have restricted the formalism to the bare minimum. A more complete - but still simplified - summary of the Botta et al. (2017a) theory is provided in appendix A. 
Still, a number of formulas appear in Sects. 2 to 6 . In the rest of this section we introduce the notation used in these formulas. This is a blend of standard mathematical notation and standard (Haskell, Idris, Agda, etc.) functional programming notation.

Thus, for instance, in Sect. 2, we write Technology $=$ \{Available, Unavailable $\}$ to posit that Technology is a set that consists of two elements: Available and Unavailable. This is plain set comprehension as in Bool $=\{$ False, True $\}$, $A=\{7,4,2\}$ or Even $=\{2 * n \mid n \in \mathbb{N}\}$.

Further, in Sect. 2, we write State $:(t: \mathbb{N}) \rightarrow$ Type to posit that "State $t$ denotes the set of states the decision maker can observe at the $t$-th decision step". This is now standard Idris notation. Idris (and Haskell, Agda) follows the usual meaning of parentheses in mathematics: to enclose a sub-expression to resolve operator precedence. The special notation $f(a)$ for the value of a function $f: A \rightarrow B$ at $a \in A$ (very often used in physics and engineering) uses parentheses in a nonstandard way.

Another possible source of confusion is the signature (type) of the function State. Its domain consists of values of type $\mathbb{N}$. These are natural numbers. But its co-domain consists of values of type Type! Thus, a legal definition of State could be

$$
\text { State } t=\text { Bool }
$$

which posits that State is the constant function that returns the type Bool for every $t$. Being able to implement functions that return types is a key feature of dependently typed languages. Among other things, it allows one to encode firstorder logic propositions as types. Thus, for instance

$$
\begin{aligned}
& \text { BoundedBy }: \mathbb{N} \rightarrow \text { List } \mathbb{N} \rightarrow \text { Type } \\
& \text { BoundedBy } n m s=\operatorname{All}(\lambda m \Rightarrow m \sqsubseteq n) m s
\end{aligned}
$$

is a legal function definition, and a value of type BoundedBy $5 x s$ is equivalent to a logical proof that all elements of $x s$ are bounded by 5. Perhaps unexpectedly, the type of $m \sqsubseteq n$ on the right-hand side of the definition of BoundedBy is Type, not Bool. This also the type of All $(\lambda m \Rightarrow m \sqsubseteq n) m s$ as can be seen from the declaration of BoundedBy. Thus, $m \sqsubseteq n$ and $A l l(\lambda m \Rightarrow m \sqsubseteq n) m s$ encode logical propositions as types: they are propositional types. Here, (足) and All are standard data types defined in the Idris libraries. Being able to encode logical propositions as types is crucial for formulating program specifications, i.e., properties that a program must satisfy to be correct. Thus, for example, a program

sqrt : Double $\rightarrow$ Double

that is meant to compute the square root of a double precision floating point number might be required to fulfill

$$
\text { sqrtSpec }:(x: \text { Double }) \rightarrow 0 \sqsubseteq x \rightarrow(\operatorname{sqrt} x) *(\operatorname{sqrt} x)=x
$$

The specification sqrtSpec is logically equivalent to the proposition "for all $x$ of type Double, if $x$ is nonnegative then the square of sqrt $x$ is equal to $x " 1$. As above, $0 \sqsubseteq x$ and $($ sqrt $x) *($ sqrt $x)=x$ are values of type Type - in contrast to $0 \leqslant x$ and $(\operatorname{sqrt} x) *(\operatorname{sqrt} x)=x$ which are Boolean values. They encode properties that depend on a specific value $x$, which means they are dependent types.

The types of sqrt and sqrtSpec formulate a well-defined, unambiguous task for the programmer. This is solved by providing implementations of sqrt and sqrtSpec that are syntactically correct and total. In this case, the implementation of sqrt is said to be verified or, equivalently, machine checked. Notice that totality plays a crucial role in this context. Only total implementations of sqrt and sqrtSpec are logically equivalent to the proposition "for all $x$ of type Double, if $x$ is nonnegative then the square of sqrt $x$ is equal to $x$ ".

Because sqrtSpec represents a property of sqrt, implementations of sqrtSpec will depend on a specific implementation of sqrt: one typically starts by implementing sqrt and then tries to prove that it is correct by implementing sqrtSpec. Implementations of sqrtSpec are typically derived from pencil-and-paper proofs (that sqrt fulfills the property encoded in the type of sqrtSpec) but dependently typed languages provide a lot of support to programmers: the Idris system can "fill in" parts of the implementation of sqrtSpec automatically.

Dependent types are also the key for expressing (perhaps non-implementable) modeling assumptions or conjectures and for formalizing domain-specific notions precisely. In Sects. 2 to 6 we will not make explicit use of propositional types. But propositional types are at the core of the theory presented in Botta et al. (2017b, a) and are extensively used there and in appendix A.

Another, perhaps unfamiliar, aspect of functional notations is currying. In mathematics, a function of $n>1$ arguments is often implicitly converted to a function that takes as a single argument one $n$-tuple. In Idris we instead use nested function application. For example, if $g$ is a general function of two arguments it has type $X \rightarrow(Y \rightarrow Z)$ or simply $X \rightarrow Y \rightarrow Z$. If we apply $g$ to just one argument $x: X$, the resulting expression $g x$ is still a function (of type $Y \rightarrow Z$ ). This function can then be applied to some value $y: Y$ to obtain the result of $g$ at $x$ and $y$, written $(g x) y$, or simply $g x y$ (because function application is leftassociative $)^{2}$.

Notice that even though we do not use propositional types in 2 to 6 , most functions there are dependently typed. Thus, for instance, in the signature of Control on page 528, the type of the second argument, State $t$, depends on the value

\footnotetext{
${ }^{1} \mathrm{~A}$ more realistic specification would require that the square of sqrt $x$ be equal to $x$ up to round-off errors, but we do not insist on these details here.

${ }^{2}$ The idea that functions of more than one variable can always be written as functions of just one variable (that return functions as result) was originally proposed in Schönfinkel (1924) and popularized by Haskell B. Curry (1958). The operation has since then been referred to as currying. Its inverse is called uncurrying.
} 
of the first argument, $t$. We say that Control is dependently typed (Norell, 2007; Brady, 2013, 2017).

Finally, the Botta et al. (2017b, a) theory applied in this paper is available in the SequentialDecisionProblems component of Botta (2016-2017). This is a git repository and it is publicly available.

\subsection{Outline}

In the next section we introduce sequential emission problems and explain what it means for sequences of emission policies to be optimal. We discuss the most important differences between deterministic (certain) problems and emission problems under uncertainty. In Sect. 3 we discuss some important traits of decision making under uncertainty. The discussion is meant to prepare the specification of the stylized emission problem presented in Sect. 4. In Sect. 5 we study the impact of the uncertainties (1)-(3) on optimal policy sequences for our stylized problem. We draw preliminary conclusions and outline future work in Sect. 6.

\section{Sequential emission problems}

As anticipated in the Introduction, in this work we study the impact of uncertainties on optimal emission polices from a control theoretical (as opposed to a game theoretical) perspective. Thus, the focus is on a single decision maker and on how uncertainties affect the questions of when global emissions shall be reduced and by how much as opposed to the question of how emission reductions can actually be implemented in a situation of mutual competition.

\subsection{Sequential emission processes}

If we focus the attention on a single decision maker and on global emissions, sequential emission problems can be described quite straightforwardly. At the core of any such problems is a sequential emission process (SEP). Informally, a sequential emission process can be described in terms of three notions.

The first notion is that of a state. A state represents the information available to the decision maker at a given decision step. Typically, the state of a decision process consists of a number of aggregated measures. These include, for instance, economic growth measures, GHG concentration measures and current emission level.

Often, the information available to the decision maker is imperfect. For instance, for a given measure, the decision maker might only be able to know a probability distribution instead of a precise value. Another possibility is that the decision maker only knows that, e.g., a GDP measure lies within certain bounds.

In the stylized sequential emission problem discussed in Sect. 4, for example, the state consists of a tuple of four values. These represent an amount of cumulated GHG emissions, an implemented emission level, a level of availability of efficient technologies for reducing GHG emissions and a state of the world. In that problem, we will assume that the decision maker can only distinguish between low and high emissions

EmissionLevel $=\{$ Low, High $\}$

and available or unavailable efficient GHG emission reduction technologies

$$
\text { Technology }=\{\text { Available, Unavailable }\}
$$

Similarly, the state of the world will be just good or bad:

$$
\text { World }=\{\text { Good, Bad }\}
$$

In realistic SEPs, decision makers typically have to select between more than two emission levels, efficient technologies for reducing GHG emission are available to certain degrees and the state of the world is slightly more multifaceted than just good or bad.

The second notion that characterizes a sequential emission process are the controls available to the decision maker. In the context of climate change studies, controls are often referred to as options, actions or policies. To avoid confusion with the notion of policy from Sect. 2.3 below, we will call them controls.

In GHG emission problems, controls are often phrased in terms of abatement levels or, equivalently, in terms of maximum GHG emissions growth rates. Thus, for instance, in Webster (2002) and over the first decision step (for the time interval between 2010 and 2019) controls can be one of eight values: $0,0.2,0.4,0.6,0.8,1.0,1.2$ and 1.4. Here, a value of 0.4 represents a maximal emissions growth rate of $0.4 \%$. In the emission problem of Sect. 4, we will further oversimplify this picture and only consider low and high GHG emissions.

Notice that, in general, not all controls are available in every state and at every decision step. In other words, the abatement levels that can be selected in a given state can depend on that specific state. Thus, in our problem from Sect. 4, we allow for the probability of implementing low (high) emissions in the next period to depend on the current emission level. As discussed in Webster (2008), the probability of implementing low (high) emission levels in the next period is higher if the current emissions are already low (high) than if the current emissions are high (low). This kind of uncertainty accounts for, among other things, the inertia of legislation and, of course, political instabilities. Thus, one can fully describe the states and the controls of a sequential decision process by defining two functions:

$$
\begin{aligned}
& \text { State }:(t: \mathbb{N}) \rightarrow \text { Type } \\
& \text { Control }:(t: \mathbb{N}) \rightarrow(x: \text { State } t) \rightarrow \text { Type }
\end{aligned}
$$

The interpretation is as follows: State $t$ denotes the set of states the decision maker can observe at the $t$-th decision 
step. Similarly, Control $t x$ represents the controls that are available to the decision maker at decision step $t$ and in state $x$. Remember that, as explained in Sect. 1.4, we denote function application by juxtaposition.

The third notion that characterizes a sequential emission process is that of a transition function. Informally, transition functions describe how states change, at each decision step, as a consequence of the controls selected by the decision maker. Thus, in a deterministic decision process the transition function has the type

$$
\begin{gathered}
\text { next }:(t: \mathbb{N}) \rightarrow(x: \text { State } t) \rightarrow(y: \text { Control } t x) \rightarrow \\
\text { State }(t+1)
\end{gathered}
$$

Again, the interpretation is that for every $t: \mathbb{N}, x:$ State $t$ and $y$ : Control $t x$, next $t x y$ is "the" new state at decision step $t+1$. Notice that the time between two successive decisions does not need to be constant. In a time-dependent decision process, for instance, there could be a function

$$
\text { time }:(t: \mathbb{N}) \rightarrow \text { Real }
$$

with time $(t+2)$-time $(t+1) \neq$ time $(t+1)$-time $t$ for all (or perhaps only for certain) values of $t$. In Webster (2002), for instance, the author investigates two-step decision problems in which the first period extends over 10 years and the second period extends over 80 years.

\subsection{Sequential emission problems}

A decision process becomes a decision problem when we fully specify the costs and the benefits that are associated with each transition. This can be done by defining a reward function. A reward function is a function that, at each decision step, associates a value with every current state, selected control and next state:

$$
\begin{aligned}
\text { reward }: & (t: \mathbb{N}) \rightarrow(x: \text { State } t) \rightarrow(y: \text { Control } t x) \rightarrow \\
& \left(x^{\prime}: \text { State }(t+1)\right) \rightarrow \text { Val }
\end{aligned}
$$

As usual, we write reward in curried form and reward t $x y x^{\prime}$ : Val denotes the reward of selecting the control $y$ in $x$ at step $t$ and ending up in $x^{\prime}$. Typically, Val is $\mathbb{R}$. An obvious question is: why shall reward explicitly depend on $x^{\prime}$ ? If $x^{\prime}=$ next $t x y$ is "the" next state, it seems that

$$
(t: \mathbb{N}) \rightarrow(x: \text { State } t) \rightarrow(y: \text { Control } t x) \rightarrow \text { Val }
$$

would be a more appropriate type for reward. The reason for including a new state $x^{\prime}$ in the signature of reward is uncertainty, as we explain in the following paragraphs. We have seen that, in deterministic decision processes, transition functions have the type

$$
\begin{aligned}
& (t: \mathbb{N}) \rightarrow(x: \text { State } t) \rightarrow(y: \text { Control } t x) \rightarrow \\
& \text { State }(t+1)
\end{aligned}
$$

What if the decision process is affected by uncertainties? If selecting an abatement level in a given state has uncertain outcomes (perhaps because of externalities or because the consequences of implementing certain emission reductions are not fully understood), it would be unsuitable to describe the decision process in terms of a transition function that returns a single next state. In this case, the transition function should return a set of possible next states or a probability distribution of next states. As detailed in Botta et al. (2017b, a), we can account for different kinds of uncertainties in decision processes with transition functions of the form

$$
\begin{aligned}
\text { next }: & (t: \mathbb{N}) \rightarrow(x: \text { State } t) \rightarrow(y: \text { Control } t x) \rightarrow \\
& M(\text { State }(t+1))
\end{aligned}
$$

where $M$ is a functor. It represents the type of uncertainties underlying the decision process. For deterministic processes, $M$ is just the identity functor: $M=I d$. For stochastic processes, $M$ represents probability distributions. This is the case considered in this work. Thus, we take $M=P r o b$ where Prob $X$ is the type of simple probability distributions ${ }^{3}$ on $X$. Therefore, next $t x y$ is a probability distribution on next states that is, a value of type Prob $($ State $(t+1))$. The states in next $t x y$ are those that can be obtained after decision step $t$ by selecting $y$ in state $x$. Thus, in a stochastic decision process, selecting a control does not yield a unique next state but a whole set of possible next states with their probabilities. Therefore, the reward function has to explicitly depend on $x^{\prime}$ because this cannot be computed from the current state $x$ and the selected control $y$ unambiguously. This justifies the signature of reward as given above.

We can summarize the results obtained so far in the observation that stochastic sequential emission problems can be specified in terms of four functions:

$$
\begin{aligned}
\text { State }: & (t: \mathbb{N}) \rightarrow \text { Type } \\
\text { Control }: & (t: \mathbb{N}) \rightarrow(x: \text { State } t) \rightarrow \text { Type } \\
\text { next }: & (t: \mathbb{N}) \rightarrow(x: \text { State } t) \rightarrow(y: \text { Control } t x) \rightarrow \\
& \text { Prob }(\text { State }(t+1)) \\
\text { reward }: & (t: \mathbb{N}) \rightarrow(x: \text { State } t) \rightarrow(y: \text { Control } t x) \rightarrow \\
& \left(x^{\prime}: \text { State }(t+1)\right) \rightarrow \text { Val }
\end{aligned}
$$

We define these functions for our stylized emission problem in Sect. 4. For the time being, we need to better understand the decision problem that four such functions specify. This is crucial for understanding the notions of policy and policy sequence introduced in the next section.

The idea is that, for a fixed number of decision steps, the decision maker seeks controls (emission levels) that maximize a sum of the rewards obtained over those steps. The emphasis here is on "a sum": depending on the specific problem at stake, future rewards might need to be discounted and the way values of type $\mathrm{Val}$ are added up might not be completely trivial. As explained in detail in Botta et al. (2017a), fully specifying stochastic SDPs requires defining State, Control, next and reward and choosing a measure for weighting uncertain outcomes. Formally, a measure is just a function that reduces probability distribution on values to values

\footnotetext{
${ }^{3}$ In a nutshell, simple probability distributions are probability distributions with finite support; see Botta et al. (2017a).
} 
meas $:$ Prob Val $\rightarrow$ Val

The expected value function is probably the most widely used measure in the study of stochastic SDPs. But other measures are possible. Depending on the specific problem and the kind of uncertainties, other measures might be more suitable than the expected value. Thus, for instance, a risk-averse decision maker might adopt a worst measure rather than relying on the expected value. It is also conceivable, that a decision maker adopts different measures of uncertainty at different decision steps. The theory summarized in appendix A can be easily extended to cope with this situation. In Sect. 4 , we walk the reader through the full specification of our stylized emission problem, including uncertainty measures.

Solving SDPs is not trivial. For this, we instantiate the generic backward induction algorithms presented in Botta et al. $(2017 \mathrm{~b}, \mathrm{a})$. We do not need to discuss these methods in detail here, but before we move to Sect. 4 , it is important to achieve a good understanding of what it means to solve a stochastic SDP and what it means for sequences of policies to be optimal.

In the rest of this section, we informally discuss the notions of policy, policy sequence and optimality of policy sequences. We do so in the context of sequential emission problems but the ideas apply to SDPs in general. In Sect. 3, we discuss a number of basic facts about sequential emission problems. These, too, apply to sequential decision problems without loss of generality.

\subsection{Emission policies}

We have pointed out that in stochastic sequential emission problems, selecting an emission (abatement) level at a given decision step and in a given state does not usually yield a unique next state. Instead, we obtain a probability distribution on next states. The distribution encodes the uncertainties associated with the decision process under study. Thus, for instance, the decision maker might select to reduce emissions by $2 \%$ but what actually gets implemented is a smaller reduction, perhaps because of political inertia or as a consequence of increased economic activity.

One consequence of uncertainties is that, even if decision makers could fix a priori an emission schedule or path ${ }^{4}$, they would not know the state obtained after a fixed number of decision steps. This is, again, because each single step yields a probability distribution on next states, not a single next state.

Thus, the best a decision maker can hope to obtain as a solution to a stochastic sequential emission problem is a sequence of rules that indicate which control (abatement level) to select for each decision step and, at that step, for each possible state.

In control theory, such "rules of action" are called policies. This is also the sense in which the word policy has been

\footnotetext{
${ }^{4}$ Strictly speaking, this is impossible because, as we have seen, feasible emissions in a given state may depend on that state.
}

used in Botta et al. (2017b, a). The control theoretical notion matches the notion of strategy in game theory quite well $(\mathrm{Fu}-$ denberg and Tirole, 1991), but notice that in plain English the term policy is ambiguous: sometimes it is used to denote a plan (course) of action and sometimes a rule of action: see www.merriam-webster.com/dictionary/policy.

Here we follow the control theory standard and policy sequences are just sequences of functions, one for each decision step. A sequence of policies for $n+1$ decision steps consists of a policy $p$ for the $t$-th decision step and of a policy sequence $p s$ for $n$ further steps. Formally we write

$$
(p:: p s) \text { : PolicySeq } t(n+1)
$$

with $p$ : Policy $t(n+1)$ and $p s:$ PolicySeq $(t+1) n$. Here, $::$ is the operator that prepends a policy to a (possibly empty) policy sequence, see appendix A and Sects. 3.5, 3.7 and 3.9 of Botta et al. (2017a). More formally, if $p s=\left[p_{1}, p_{2}, p_{3}\right]$ then $p:: p s=\left[p, p_{1}, p_{2}, p_{3}\right]$ for all $p:$ Policy $t(n+1)$, ps : PolicySeq $(t+1) 3$.

But what does it mean for a sequence of emission policies to be optimal? The decision maker aims at maximizing the sum of rewards over a fixed number of steps. Thus, $(p:: p s)$ is "an" optimal policy sequence for $n+1$ decision steps if and only if no other sequence attains a higher sum of rewards (over $n+1$ steps) for any given $x$ : State $t$.

While fairly intuitive, formalizing this notion of optimality is not completely trivial. This is because, in a stochastic emission problem, a selected abatement level does not entail a unique next state, as explained above. Thus, for any possible next state (and therefore for any possible value of taking $n$ further decision steps with the policies of $p s$ and starting from that state) we have a corresponding reward and a probability. Such a probability distribution of rewards has to be measured with meas in order to obtain the value of making $n+1$ decision steps according to the policy $p$ and the policy sequence $p s$.

In appendix A, we discuss the computation of the value of policy sequences in detail. In order to get an intuition of the notion of optimality for policy sequences, it is sufficient to recognize that one can precisely define a function

$$
\text { val }:(x: \text { State } t) \rightarrow \text { PolicySeq } t n \rightarrow \text { Val }
$$

In the theory of SDPs, val is called the value function. As one would expect, val $x$ ps is the value, in terms of the measured sum of possible rewards, of performing $n$ decision steps with the policy sequence $p s$ and starting in state $x$. Crucially, val $x$ ps only depends on State, Ctrl, next, reward, meas and the rule for adding up rewards.

The value function allows us to give a precise meaning to the intuitive notion of optimality of policy sequences discussed above. More importantly, it allows us to actually compute optimal sequences of policies, at least for decision problems that fulfill certain natural conditions.

Again, a comprehensive discussion of the notion of optimality and the conditions under which optimal policy se- 
quences can be computed goes well beyond the scope of this work. We refer the interested reader to appendix A and to Botta et al. (2017a) and close this section by recalling an often neglected fact on decision making under uncertainty.

A fundamental difference between decision making under deterministic transition functions and decision making under uncertainty is that, in the latter case, regret cannot generally be avoided. Here, by regret we mean a judgment in hindsight often triggered by an unlucky sequence of transitions. Thus, for instance, a system for optimal routing may recommend that a driver leave a highway in order to avoid an upcoming traffic jam. On the alternative road, the driver may get involved in a car accident and finally regret having left the highway. Of course, the driver's regret does not change the fact that leaving the highway was a best choice (under the problem's reward function, measure of possible rewards, etc.) at the point in time in which the choice had to be made.

In both the deterministic and in the uncertain case, the notion of "best decision" is the same: at, for example, decision step $t$ and in $x$ : State $t$, a best decision $y^{*}: \operatorname{Ctrl} t x$ is a decision that cannot be bettered (in terms of sum of possible rewards) given the decision problem (that is, the functions State, Ctrl, next and reward, the measure meas and the rule for adding rewards) and a sequence of policies (optimal or not) for taking $n$ further decisions.

But when the outcome of a decision step is a probability distribution on next states, we will have many possible trajectories of length $n+1$ starting in $x$ instead of just one. In general, there is nothing preventing some of these trajectories from containing states that make any best decision in $x$ regrettable. This is true even for trajectories of length 1 , which is for $n=0$.

\section{Logical consequences of SEPs}

In this section we discuss some logical consequences of the notions introduced in 2. A first consequence of the notion of optimal policy sequence is that optimal decisions may vary over time: a best control at a given step does not need to be a best control at a subsequent (or previous) step even if the decision maker observes the same state at both decision steps. There is nothing worrying in this fact: the possible time inconsistency of optimal policies and Bellman's principle of optimality (Bellman, 1957) are perfectly consistent!

Another consequence of the notions introduced in Sect. 2 is that exploiting available information is crucial in decision making under uncertainty. We have seen that, under uncertainty, regret cannot in general be avoided. In spite of this fact, the notion of optimal policy sequence and of "best" decision are both clear and compelling: optimal policy sequences for SEPs provide decision makers with rules for selecting emission levels that, at any decision step, cannot be bettered given the information available to the decision maker at that step.
The crucial point is exploiting the information available at a given decision step. As seen in Sect. 2, this information is coded in the notion of State and the mechanisms for exploiting such information are policies or action rules. Taking decisions on the basis of optimal policies is in most cases better than selecting controls according to fixed (ex ante) action plans. This is because, in contrast to fixed action plans, policies provide an action for every possible state that can eventually be reached (ex post) at a given decision step. They account for all the information available to the decision maker at that step. Further, optimal policies entail actions that cannot be bettered.

In Sect. 5, we discuss optimal policies for the emission problem of Sect. 4. Because these policies are computed using the verified framework presented in Botta et al. (2017b, a), we know (in spite of the uncertainties affecting emission problems, for certain!) that the conclusions that we draw for our uncertain emission problem are logical consequences of the problem specification. Computing optimal policies with a verified implementation is crucial because, in contrast to other properties of solutions to computational problems, optimality cannot in general be established by testing. This is a well-known case in which proving is difficult but still easier than testing; see Ionescu and Jansson (2013).

A third logical consequence of the notions introduced in the previous section is that best controls and optimal policies are not generally unique. In Sect. 5 we discuss a problem setup in which both increasing and decreasing emissions are optimal. When applying optimal control to inform policy advice and decision making, it is important to keep in mind that optimal policies are not necessarily unique: different optimal emission sequences can yield different sets of possible emission paths. Decision makers might not be able to distinguish them in terms of measures of possible sums of rewards, but they still might have reasons to prefer certain optimal emission policies to others. For instance, precautionary approaches might lead decision makers to prefer optimal policies that entail low risk levels to high risk optimal policies.

Another logical consequence of decision making under uncertainty is that the value of policies depends not only on the problem-specific reward function and on the way rewards are added (e.g. via discounting) but also on how the decision maker weights uncertain outcomes. This is captured by the measure function meas. Different measures reflect different attitudes or dispositions, e.g., towards risk.

As explained in Ionescu (2009), decision makers are free to choose whatever measure they like as far as it fulfills a monotonicity condition. Informally, this condition says that if one increases the $\mathrm{Val}$ values of a probability distribution by any arbitrary amount (leaving their probabilities unchanged), its measure shall not decrease (see appendix A). The expected value, in much the same way as worst and best case measures, fulfill this condition. But notice that, as shown at pp. 112-116 of Ionescu (2009) in the context of a formal- 
ization of vulnerability notions, measures that pick up the most (least) probable $\mathrm{Val}$ value of a probability distribution do violate the monotonicity condition. It is a responsibility of scientific advisors to make sure that decision making is informed by meaningful, monotonic measures.

\section{A stylized sequential emission problem}

In this and in the next section, we study how optimal sequences of GHG emission policies are affected by 1) uncertainty about the implementability of decisions on GHG emission reductions, 2) uncertainty about the availability of efficient technologies for reducing GHG emissions and 3) uncertainty about the implications of exceeding a critical threshold of cumulated GHG emissions.

As anticipated in the Introduction, we first specify a stylized sequential emission problem that accounts for all three sources of uncertainty and yet is simple enough to support an investigation of the logical consequences of different assumptions on such uncertainties. In Sect. 5 we discuss the optimal policies obtained for our stylized problem under different assumptions.

We specify our stylized emission problem by instantiating the theory for SDPs summarized in appendix A. Technically, this is done by defining all the undefined variables (filling the holes) in the modules that implement the theory. For the implementation provided in the SequentialDecisionProblems component of Botta (2016-2017), these are the holes in

- CoreTheory, FullTheory, Utils;

- CoreTheoryOptDefaults, FullTheoryOptDefaults, FastStochasticDefaults;

- TabBackwardsInduction; and

- TabBackwardsInductionOptDefaults.

For a detailed discussion on how to specify an SDP, see Botta et al. (2017a). A complete implementation of our specification is available in applications/EmissionsGame2. In the rest of this section, we skip most technical details and focus on the specification of the emission problem from an applicational perspective.

As anticipated in the Introduction, we specify our stylized emission problem as a stochastic SDP. Thus, $M=$ Prob. We have to define the four functions State, Control, next and reward introduced in Sect. 2. We start by defining the controls, i.e., the options available to the decision maker.

\subsection{Controls}

In our stylized emission problem, at each decision step the decision maker can only select between low and high GHG emissions. Thus,

Control $t x=$ LowHigh where LowHigh is a type inhabited by only two values: Low and High. The idea is that low emissions, if actually implemented, increase the cumulated GHG emissions less than high emissions.

\subsection{States}

At each decision step, the decision maker has to choose between low and high emission levels on the basis of four values: a measure of cumulated GHG emissions, the current emission level (itself either low or high), the availability of effective technologies for reducing GHG emissions and a "state of the world". Effective technologies for reducing GHG emissions can be either available or unavailable. The state of the world can be either good or bad:

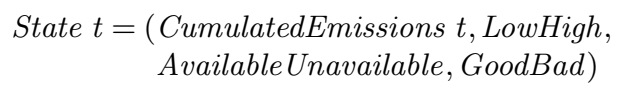

The idea is that the decision process starts with zero cumulated emissions, high emission levels, unavailable GHG technologies and with the world in a good state. In these conditions, the probability for the world to turn to the bad state is low. But if the cumulated emissions increase beyond a fixed critical threshold, the probability that the world becomes bad increases. If the world is in the bad state, there is no chance to come back to the good state. Similarly, the probability that effective technologies for reducing GHG emissions will become available increases after a fixed number of decision steps. Once available, effective technologies stay available forever.

In a realistic problem, the capability of actually implementing a decision on a given GHG emission level typically depends on a variety of factors. In our stylized problem, we follow Webster $(2002,2008)$ and focus on the uncertainties about the implementability of decisions on GHG emission reductions that come from inertia: implementing low emissions is easier when low emission measures are already in place than when the current emissions are high. Similarly, implementing high emission measures is easier if the current emissions are high than under low emissions regulations.

\subsection{Transition function}

We have defined State $t$ to be a tuple of values representing cumulated GHG emissions, the current emission level, the availability of effective technologies for reducing GHG emissions and the state of the world at decision step $t$. As our stylized emission problem is stochastic, its transition function at decision step $t$ yields a probability distribution on values of type State $(t+1)$.

The idea is that low emission levels leave the cumulated emissions unchanged and high emissions increase the cumulated emissions. Without loss of generality, we can take such an increase to be 1 . We have mentioned that the probability 
of the state of the world to become bad depends on a critical cumulated emissions threshold. Let's call this threshold $\operatorname{cr} E$

$$
\text { crE : Double }
$$

and let $p S 1$ and $p S 2$ represent the probabilities of staying in a good world when the cumulated emissions are smaller than or equal to $\operatorname{cr} E$ and greater than $c r E$, respectively:

$$
\text { pS1: NonNegDouble }
$$$$
\text { pS2 : NonNegDouble }
$$

Thus, the probabilities of getting into a bad world below and above the threshold are $1-p S 1$ and $1-p S 2$, respectively. As a sanity check, we require $p S 2$ to be less than or equal to $p S 1$.

Next, we have to specify the uncertainties about the availability of efficient technologies for reducing GHG emissions. This, too, can be done in terms of a critical number of decision steps

$$
\operatorname{crN}: \mathbb{N}
$$

and of two probabilities: the probability of effective technologies for reducing GHG emissions becoming available when the number of decision steps is below or at $\operatorname{crN}$ and the probability for the case in which $t$ is above $c r N$ :

$$
\begin{aligned}
& p A 1: \text { NonNegDouble } \\
& \text { pA2 : NonNegDouble }
\end{aligned}
$$

Also for these probabilities we need a sanity check: $p A 1$ shall be at most equal to $p A 2$. Finally, we have to specify the uncertainties about the implementability of decisions on GHG emission reductions. Following the discussion in the previous section, we do so in terms of four conditional probabilities. These are the probability of implementing low emission measures when the current emissions measures are low and low emissions are selected $p L L$, the probability of implementing low emission measures when the current emissions measures are high and low emissions are selected $p L H$ and their counterparts for high emissions:

$$
\begin{aligned}
& p L L: \text { NonNegDouble } \\
& p L H: \text { NonNegDouble } \\
& p H L: \text { NonNegDouble } \\
& p H H: \text { NonNegDouble }
\end{aligned}
$$

Also for these probabilities, we require two sanity checks to be fulfilled: $p L H$ shall not exceed $p L L$ and $p H L$ shall not exceed $p H H$. With these parameters in place, the transition function next can be implemented by cases. For a full implementation, we refer the reader to applications/EmissionsGame2. As an example, we discuss here the case in which the current state is

$$
x=(e, \text { High, Unavailable, Good })
$$

the decision maker has opted for low emissions, $e$ is smaller than or equal to $\operatorname{cr} E$ and $t$ is smaller than or equal to $c r N$. In this case, the result of next $t x$ Low is a probability distribution with the following assignments:

$$
\begin{aligned}
& (e, \quad L, U, G) \Rightarrow \quad p L H *(1-p A 1) * \quad p S 1 \\
& (e+1, H, U, G) \Rightarrow(1-p L H) *(1-p A 1) * \quad p S 1 \\
& (e, \quad L, A, G) \Rightarrow p L H * p A 1 * p S 1 \\
& (e+1, H, A, G) \Rightarrow(1-p L H) * \quad p A 1 * \quad p S 1 \\
& (e, \quad L, U, B) \Rightarrow \quad p L H *(1-p A 1) *(1-p S 1) \\
& (e+1, H, U, B) \Rightarrow(1-p L H) *(1-p A 1) *(1-p S 1) \\
& (e, \quad L, A, B) \Rightarrow p L H * p A 1 *(1-p S 1) \\
& (e+1, H, A, B) \Rightarrow(1-p L H) * \quad p A 1 *(1-p S 1)
\end{aligned}
$$

Here $L, H, U, A, G$ and $B$ are aliases for Low, High, Unavailable, Available, Good and Bad, respectively. Notice that the marginal probability of the new state to enter a bad world is $1-p S 1$, as one would expect. Similarly, the probability of effective technologies for reducing GHG emissions becoming available is $p A 1$ (we are considering the case $t \leqslant c r N$ ) and the probability of implementing low emission measures is $p L H$ as the current emission levels are high. Similar assignments hold for $x=(e, H, U, B)$, $x=(e, H, A, G)$, etc.

\subsection{Reward function}

To complete the specification of our stylized emission problem, we have to define the reward function and the measure

$$
\text { meas }: \text { Prob Val } \rightarrow \text { Val }
$$

according to which the decision maker weights uncertain outcomes. Unless stated otherwise, we will take $\mathrm{Val}$ to be NonNegDouble (nonnegative, double precision, floating point numbers) and meas to be the expected value function. In this section we focus attention on the reward function

$$
\begin{aligned}
\text { reward }: & (t: \mathbb{N}) \rightarrow(x: \text { State } t) \rightarrow(y: \text { Control } t x) \rightarrow \\
& \left(x^{\prime}: \text { State }(t+1)\right) \rightarrow \text { Val }
\end{aligned}
$$

The idea is that being in a good world yields one unit of benefits per step and being in a bad world yields lower benefits. We can formalize this idea by introducing a dimensionless number

\section{$b$ : NonNegDouble}

which represents the ratio between the step benefits in a bad world and the step benefits in a good world. It goes without saying that a constant ratio is a very crude approximation that can only be justified in a stylized problem. In sequential emission problems aiming at informing decision making under realistic conditions, the costs and the benefits of not transgressing global emission thresholds are likely to be time dependent and have to be carefully estimated, e.g., by running global climate models coupled with economic models and perhaps energy models. Unless otherwise stated, we will take $b$ to be equal to 0.5 . Of course, we require the $b$ ratio to be smaller than or equal to 1 . 
Emitting GHGs also brings step benefits, e.g. by supporting economic growth. These can be represented as a fraction of the step benefits of being in a good world. Moreover, low emissions bring lower benefits (higher costs) than high emissions and reducing emissions when effective technologies are unavailable costs more than reducing emissions when such technologies are available. We can summarize this state of affairs in terms of three dimensionless numbers. A first number represents the ratio between the step benefits of low emissions and the step benefits in a good world when effective technologies for reducing GHG emissions are unavailable

$$
\text { lu : NonNegDouble }
$$

A second number represents the same ratio when effective technologies are available

$$
\text { la: NonNegDouble }
$$

Finally, a third number represents the ratio between the step benefits obtained through high emissions and the step benefits in good worlds

$$
h: \text { NonNegDouble }
$$

We require $l u, l a$ and $h$ to be smaller than or equal to $1, l u$ to be smaller than or equal to $l a$ and the latter to be smaller than or equal to $h$. With these notions in place, we can easily implement the reward function of our stylized emission problem. The idea is that the rewards only depend on the next state (the state during the period starting with the current decision) and not on the current state or on the selected control. We have eight cases with the following assignments

$$
\begin{aligned}
& (e, H, U, G) \Rightarrow 1+h \\
& (e, H, U, B) \Rightarrow b+h \\
& (e, H, A, G) \Rightarrow 1+h \\
& (e, H, A, B) \Rightarrow b+h \\
& (e, L, U, G) \Rightarrow 1+l u \\
& (e, L, U, B) \Rightarrow b+l u \\
& (e, L, A, G) \Rightarrow 1+l a \\
& (e, L, A, B) \Rightarrow b+l a
\end{aligned}
$$

To sum up, the parameters that define the reward function of our stylized emission problem, their default values and sanity constraints are collected in Table 1.

Completing the specification of our problem and computing optimal sequences of emission policies requires filling in some more details. These are annotated and discussed in applications/EmissionsGame2.

They are pertinent to the notions of reachability, viability, finiteness and decidability. These notions are crucial for understanding the problem of computing optimal policies under uncertainty but their discussion would go well beyond the scope of this work. We refer the interested reader to Botta et al. (2017a).

\begin{tabular}{|l|r|l|}
\hline parameter & value & constraint \\
\hline$b$ & 0.5 & $b \leqslant 1$ \\
\hline$h$ & 0.3 & $h \leqslant 1$ \\
\hline$l a$ & 0.2 & $l a \leqslant h$ \\
\hline$l u$ & 0.1 & $l u \leqslant l a$ \\
\hline
\end{tabular}

Table 1. Reward function parameters, default values and constraints

\section{Optimal policies}

In this section we discuss optimal emission policies for the stylized emission problem of Sect. 4 and study the impact of uncertainties (1)-(3) on such policies. As explained in Sect. 3 , the computed policies have been machine checked to be optimal. Thus, they only depend on our problem specification. This is simple enough to allow for the deduction some general properties that optimal decisions - decisions taken according to optimal policy sequences - have to fulfill.

A first one is that no optimal policy sequence can require the selection of low emissions when the state of the world is bad. This is because, as posited in Sect. 4, there is no way to make a transition from a bad world to a good world and, in a bad world in much the same way as in a good world, higher emissions bring more emission benefits. In other words, reducing emissions can only pay off if it makes it possible (albeit not certain) to avoid transitions to a bad world, if perhaps only for a limited number of steps. Once such a transition has taken place, reducing emissions is pointless. A consequence of this fact is that in the last step it is always optimal to select high emissions. In a realistic emission problem, one could easily prevent this situation by introducing a suitable "unsustainability" penalty in the reward function at the last decision step.

We do not need to deal with such complications here but it is perhaps useful to point out that very often, seemingly natural and innocuous assumptions (in this case, that the number of decision steps is finite and known to the decision maker) can have nontrivial consequences for "best" decisions. Thus, for instance, the rate at which rewards are discounted in integrated assessment models of climate change typically has a severe impact on optimal emission policies. Thus, in policy advice, it is crucial to apply theories that require all assumptions to be made explicit. This was one of the guiding criteria in developing the theory of policy advice and avoidability discussed in Botta et al. (2017a).

Unless specified, we consider nine decision steps with $c r E=4$ and $c r N=2$. Thus, it takes at least five decision steps (and five periods with high emissions) to achieve states in which the sum of the cumulated emissions exceeds $c r E$, and therefore the probability of a transition to a bad world increases from $p S 1$ to $p S 2$. Similarly, with $c r N=2$, it takes three decision steps to achieve states in which the probability that effective technologies for reducing GHG emissions will become available increases from $p A 1$ to $p A 2$. 
In other worlds, if $p S 1=p A 1=0$ and $p S 2=p A 2=1$, effective technologies will be available (with certainty) after four decision steps. And after five periods of high emissions, a transition to a bad world will occur. This is the deterministic base case studied in the next section.

\subsection{The deterministic base case}

Before studying the impact of uncertainties on optimal policies, we consider the certain case. Beside $p S 1=p A 1=0$ and $p S 2=p A 2=1$ we also have $p L L=p L H=p H L=$ $p H H=1$. Thus, there is no uncertainty about the implementability of emission measures: decisions on reducing or increasing emissions are implemented with probability 1.

Notice that the complete absence of uncertainties implies that, for any initial state and policy sequence (optimal or not) there is exactly one possible state-control trajectory: namely, that determined by that policy sequence. Thus, for instance, if we start in $(0, H, U, G)$ (zero cumulated emissions, high emissions, unavailable efficient technologies and a good world) and adopt the policy of constantly increasing emissions, we obtain the state-control trajectory

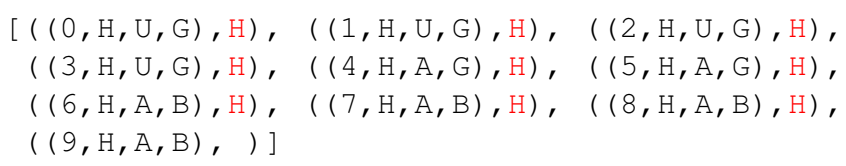

with probability 1 . The sum of rewards associated with this "certain" trajectory is 9.7: these result from five periods in a good world (step benefits equal to 1), four periods in a bad world (step benefits 0.5 ) and nine periods of high emissions (emission benefits per step of 0.3). As expected, efficient technologies for reducing GHG emissions become available at decision step four (after four decisions) and the transition to a bad world takes place after five periods of high emissions and six decisions. We can do a bit better by selecting low emissions at every step. In this case the state-control trajectory is

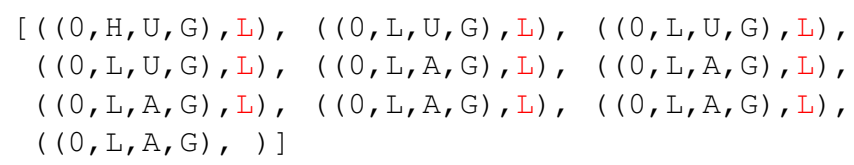

What are optimal policy sequences like in the certain case? The intuition is that, in at least four decision steps, emissions should be high. This yields higher rewards at no risk of getting into a bad world. One would also expect that lower emissions are selected (and implemented with certainty) in states in which efficient technologies for reducing GHG emissions are available. The trajectory associated with an optimal sequence of policies

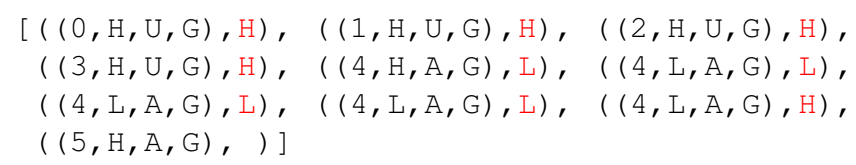

shows that such intuition is correct. The sum of rewards associated with this trajectory is 11.3 . By selecting low emissions starting from the fifth decision step, the optimal policy guarantees that the world stays in the good state. At the last decision step, high emissions are selected, as anticipated.

The computation supports the intuition that, in a world without uncertainties, it is best to delay emission reductions until efficient technologies become available. Of course, this requires knowing the critical number of decision steps $c r N$.

\subsection{The impact of uncertainties about the implementability of decisions on emission reductions.}

What happens to optimal policies if we factor in uncertainties about the implementability of decisions on emission reductions or increases?

Let's consider the case in which the probability of implementing low emission measures in the next period is higher if the current emissions are already low than in the case in which the current emissions are high. Conversely, the probability of implementing high emissions in the next period is higher if the current emissions are high. In other words, we have $p L H<p L L$ and $p H L<p H H$ instead of $p L L=p L H=$ $p H L=p H H=1$. Specifically, consider optimal policies for the case $p L L=p H H=0.9$ and $p L H=p H L=0.7$.

Our decision problem is now no longer deterministic. Thus, a policy (optimal or not) entails a whole set of possible future state-control trajectories. More precisely, we have $2^{9}=512$ possible trajectories: we take nine decision step and, at every decision step and no matter whether we select low or high emissions, we have two possible outcomes. Now, the "business as usual" policy of always selecting high emissions yields the trajectory

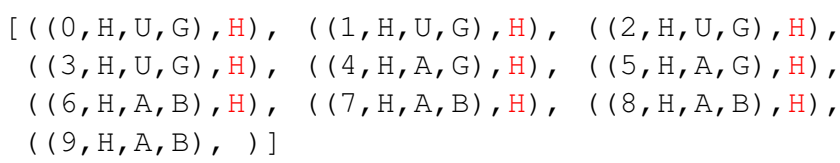

with probability $0.9^{9} \approx 0.387$. The two next most likely trajectories are

$$
\begin{array}{lll}
{[((0, H, U, G), H),} & ((1, H, U, G), H), & ((2, H, U, G), H), \\
((3, H, U, G), H), & ((4, H, A, G), H), & ((5, H, A, G), H), \\
((6, H, A, B), H), & ((7, H, A, B), H), & ((8, H, A, B), H), \\
((8, L, A, B), ~)] &
\end{array}
$$

and

$$
\begin{array}{lll}
((0, H, U, G), H), & ((1, H, U, G), H), & ((2, H, U, G), H), \\
((3, H, U, G), H), & ((4, H, A, G), H), & ((4, L, A, G), H), \\
((5, H, A, G), H), & ((6, H, A, B), H), & ((7, H, A, B), H), \\
((8, H, A, B),)] &
\end{array}
$$

with probabilities of 0.043 and 0.033 . The expected sum of rewards (remember that meas is the expected value function) is 9.904. The computed optimal policies for the same problem yield the trajectory 


$$
\begin{array}{lll}
((0, H, U, G), H), & ((1, H, U, G), H), & ((2, H, U, G), L), \\
((2, L, U, G), L), & ((2, L, A, G), L), & ((2, L, A, G), L), \\
((2, L, A, G), H), & ((3, H, A, G), H), & ((4, H, A, G), H), \\
((5, H, A, G),)] &
\end{array}
$$

with probability 0.234 . The two next most likely trajectories are

$$
\begin{aligned}
& {[((0, H, U, G), H),((1, H, U, G), H),((2, H, U, G), L),} \\
& ((3, H, U, G), L),((3, L, A, G), L),((3, L, A, G), L), \\
& ((3, L, A, G), L),((3, L, A, G), H),((4, H, A, G), H), \\
& ((5, H, A, G),)] \\
& {[((0, H, U, G), H),((1, H, U, G), H),((2, H, U, G), L),} \\
& ((2, L, U, G), L),((2, L, A, G), L),((2, L, A, G), L), \\
& ((2, L, A, G), H),((2, L, A, G), H),((3, H, A, G), H), \\
& ((4, H, A, G),)]
\end{aligned}
$$

both with probability 0.078 . The expected sum of rewards is 11.085. Notice that, under uncertainties about the implementability of decisions on emission reductions or increases, optimal policies dictate more precautious best decisions: instead of waiting for efficient technologies for reducing GHG emissions to become available, optimal decision making requires starting to reduce emissions after only two decision steps.

The fact that higher uncertainties about the implementability of decisions on emission reductions or increases lead to more precautionary optimal policies is confirmed by computing optimal policies for the case $p L L=p H H=0.7$ and $p L H=p H L=0.5$. In this case optimal policies dictate low emissions in the first decision steps for the three most likely possible trajectories. This is still true in the limit $p L L=$ $p H H=0.5+\epsilon$ for $\epsilon>0, \epsilon \longrightarrow 0$ although the advantage of optimal policies against nonoptimal policies (e.g., "business as usual" policies) in terms of expected rewards tends to zero as $\epsilon$ goes to zero.

In the limit case in which the decision maker has no power to enforce emission decisions for the next period and $p L L=p H H=p L H=p H L=0.5$, any policy sequence is optimal, as one would expect. As discussed in Sect. 3, this is an example of nonuniqueness of optimal policies.

\subsection{The impact of uncertainties about the availability of efficient technologies for reducing GHG emissions}

What if the probability of efficient technologies becoming available after three decision steps is less than 1 and there is a small but nonzero probability that such technologies will become available before three decision steps?

With the same uncertainties as in Sect. 5.2 $(p L L=p H H=$ 0.9 and $p L H=p H L=0.7$ ) and with $p A 1$ and $p A 2$ equal to 0.1 and 0.9 instead of 0 and 1 , we now have $2^{n} *(n+1)$ possible trajectories ${ }^{5}$ for $n$ decision steps. Thus, for $n=9$, we

\footnotetext{
${ }^{5}$ At each decision step, a possible state in which efficient technologies are not available, a $U$ state, entails four possible next states: two in which efficient technologies are available and two in
}

have 5120 trajectories instead of just 512 . The "business as usual" policy of always selecting high emissions yields the same most likely trajectory and a slightly higher expected sum of rewards: 9.91. The computed optimal policies also yield the same most likely trajectories as in Sect. 5.2 although with lower probabilities. The expected sum of rewards is 11.102 .

Thus, perhaps surprisingly, uncertainties on the availability of efficient technologies for reducing GHG emissions have little impact on optimal decisions, at least when compared to the impact of uncertainties about the implementability of decisions on emission reductions.

\subsection{The impact of uncertainties about the implications of exceeding a critical threshold of cumulated GHG emissions.}

So far we have assumed that if the critical cumulated GHG emissions threshold $\operatorname{crE}$ was exceeded, the world would turn to a bad state with probability 1 . Conversely, for cumulated emissions below the $\operatorname{cr} E$, the probability of a transition into a bad world was zero.

What if we assume a $10 \%$ probability of turning to a bad world for cumulated emissions below the $\operatorname{crE}$ and a $10 \%$ chance of staying in a good world above the critical threshold?

Adding these uncertainties to the certain "base" case yields 10 possible trajectories. These correspond to transitions to a bad world in the first through ninth decision step. In this scenario, always selecting high emissions yields the trajectory of the certain case

$$
\begin{array}{lll}
{[((0, H, U, G), H),} & ((1, H, U, G), H), & ((2, H, U, G), H), \\
((3, H, U, G), H), & ((4, H, A, G), H), & ((5, H, A, G), H), \\
((6, H, A, B), H), & ((7, H, A, B), H), & ((8, H, A, B), H), \\
((9, H, A, B), ~)] &
\end{array}
$$

with probability 0.531 . The expected sum of possible rewards is lower than in the certain case: 9.076. Similarly, optimal policies under uncertainty about the implications of exceeding $\operatorname{cr} E$ yield the possible trajectory

$$
\begin{array}{lll}
{[((0, H, U, G), H),} & ((1, H, U, G), H), & ((2, H, U, G), H), \\
((3, H, U, G), H), & ((4, H, A, G), L), & ((4, L, A, G), L), \\
((4, L, A, G), L), & ((4, L, A, G), L), & ((4, L, A, G), H), \\
((5, H, A, G),)] &
\end{array}
$$

with probability 0.387 . In the certain case, this was also the (certain) optimal trajectory. The expected sum of possible rewards is 9.731, which is much lower than in the certain case but still better than for the "business as usual" policies.

which they are not. A possible state in which efficient technologies are available (an $A$ state) only entails two possible next states because once technologies become available they stay available in all possible future states. Thus, after one decision step, we have two possible $U$ states and two possible $A$ states. After two decision steps, we have four possible $U$ states and eight possible $A$ states. After three decision steps we have eight possible $U$ states and 24 possible $A$ states. And so on. 
These results suggest that, as for the case of uncertainties about the availability of efficient technologies, uncertainties about the implications of exceeding $\operatorname{crE}$ do not affect optimal policies substantially: the intuition that lower emissions should be selected (and implemented with certainty) in states in which efficient technologies for reducing GHG emissions are available still holds.

Adding uncertainties about the implications of exceeding $c r E$ on top of uncertainties about the implementability of decisions and uncertainties about the availability of efficient technologies also does not substantially change the understanding obtained in Sects. 5.2 and 5.3. But it brings some new unexpected results.

With $p L L=p H H=0.9, p L H=p H L=0.7, p A 1=0.1$, $p A 2=0.9$ and $p S 1=0.9, p S 2=0.1$ obtains 51200 possible trajectories. For "business as usual" policies, the most likely is the usual

$$
\begin{array}{lll}
{[((0, H, U, G), H),} & ((1, H, U, G), H), & ((2, H, U, G), H), \\
((3, H, U, G), H), & ((4, H, A, G), H), & ((5, H, A, G), H), \\
((6, H, A, B), H), & ((7, H, A, B), H), & ((8, H, A, B), H), \\
((9, H, A, B),)] &
\end{array}
$$

with probability 0.135 . Remember that in the absence of uncertainty about the implications of exceeding $c r E$, the three most likely trajectories were

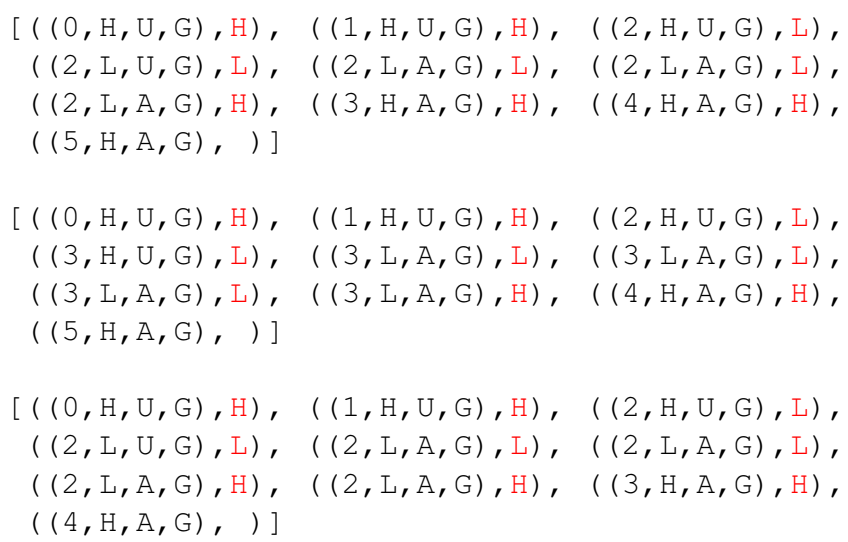

with associated rewards $11.2,11.3$ and 11.1 and probabilities $0.154,0.051$ and 0.051 . The expected sum of possible rewards was 11.102 . Adding $10 \%$ uncertainty about the implications of exceeding $c r E$ yields

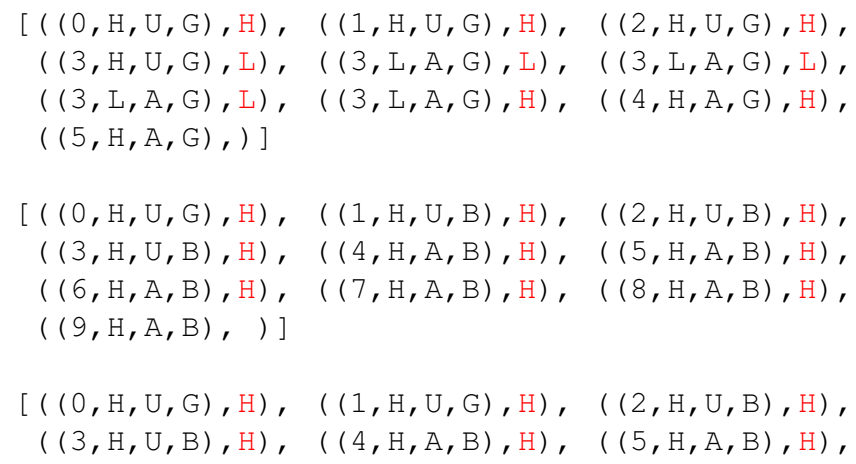

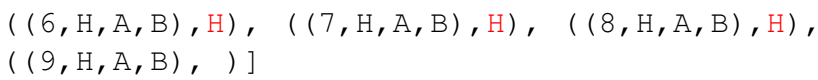

with expected rewards 11.3, 7.2 and 7.7 and probabilities $0.059,0.025$ and 0.023 , respectively. The expected sum of possible rewards is 9.543 . Now, optimal policies for the most likely trajectory require postponing emission reductions by one step: low emissions are selected starting from $t=3$ instead of $t=2$.

Notice that the optimal policies require constant high emissions both for the second and for the third most likely trajectories! This is because in these trajectories the world enters a bad state right after the first decision step (second trajectory) or after the second decision step (third trajectory). Indeed, the rewards associated with the second and the third trajectories (7.2 and 7.7, respectively) are significantly lower than the rewards associated with the most likely trajectory (11.3)

Notice also that, even though the probability of transition into a bad world is only 0.1 for cumulated emissions below $c r E$, the trajectory that entails such a transition immediately after the first decision step (the second one) is more likely to occur than the trajectory in which the world stays in the good state for the first period (third one).

This seems at the first sight counterintuitive. But it can easily be verified by inspection ${ }^{6}$ and is in fact easily explained: the crucial point is that the probability of entering a bad world at the first decision step (and then necessarily staying in a bad world) is 0.1 . By contrast, the probability of staying in a good world for one period and then getting into a bad world is, ceteris paribus, $0.9 * 0.1$. This difference makes the second trajectory more likely than the third one. Of course, both trajectories are much less likely than the first one as in the cases discussed in Sects. 5.2 and 5.3.

\section{Conclusions}

We have studied the impact of uncertainties about 1) the implementability of decisions on emission reductions, 2) the availability of technologies for reducing emissions and 3) the implications of exceeding a critical threshold of cumulated emissions on optimal emission policies in a stylized sequential emission problem.

In a nutshell, the results presented in Sect. 5 support the conclusion that uncertainties about the implementability of decisions on emission reductions (or increases) call for more precautionary policies. By contrast, uncertainties about the implications of exceeding critical cumulated emission thresholds tend to make precautionary policies suboptimal.

More specifically, the results of Sect. 5 suggest that uncertainties about the implementability of decisions on emission

\footnotetext{
${ }^{6}$ Given the probabilities $p S 1, p S 2, p A 1, p A 2, p L L, p L H$, $p H L$ and $p H H$ as above, the probability of a given trajectory is just the product of the probabilities of the corresponding transitions.
} 
reductions and, to a more limited extent, uncertainties about the implications of exceeding critical cumulated emission thresholds have a greater impact on optimal emissions policies than uncertainties on the availability of effective technologies for reducing GHG emissions.

This is at the first glance perhaps a bit surprising but actually quite understandable: if decisions on emissions (no matter whether reductions or increases) can be implemented with certainty, it is obviously better to delay necessary but costly reductions until available technologies make abatements cheaper. This holds as far as delays do not lead global emissions to exceed the critical threshold $\operatorname{cr} E$.

But if we cannot be sure that future decisions will be implemented with certainty - for instance, because of inertia in legislation or political instability - than starting to implement emission reductions (or trying to do so) sooner yields higher rewards. This is a typical case in which precautionary policies are optimal.

How much earlier it is optimal to undertake costly abatement steps (rather than waiting for technological innovation to make emission reductions cheaper) very much depends on the rewards structure and on the uncertainties of the specific emission problem at stake.

Perhaps more surprisingly, the results of Sect. 5.4 suggest that the optimal time for starting to reduce emissions also depends on the level of uncertainty about the implications of trespassing critical thresholds of cumulated emissions. As these uncertainties increase, precautionary policies become suboptimal. In other words, the better we can estimate the consequences of exceeding critical thresholds, the more it pays off to adopt precautionary policies.

Two caveats are in order here. First, while the results presented in Sect. 5 are rigorous (the optimal emission policies our conclusions rely upon have been machine checked), the stylized emission problem for which we have computed such policies is defined in terms of a small but not empty set of parameters. In particular, the value of policy sequences (optimal or not) crucially depends on the problem rewards, which means on the values of the four parameters $b, h, l a$ and $l u$; see Table 1 at the end of Sect. 4. Are our conclusions only valid for these specific values?

Apart from substantiating our findings with a careful (but necessarily prohibitively expensive) sensitivity analysis, we can try to achieve a better analytical understanding of the impact of the above parameters on optimal policy sequences.

From the definition of the reward function given at the end of Sect. 4, we can immediately deduce that at each decision step the costs of selecting low emissions are greater than or equal to

$$
h-l a
$$

Remember that $l u$ is the ratio between the benefits of low emissions and the benefits of being in a good world when effective technologies for reducing GHG emissions are unavailable. Similarly, $l a$ is the ratio between the benefits of low emissions and the benefits of being in a good world when effective technologies are available. As summarized in Table 1 , we require $l u$ to be smaller than or equal to $l a$ (effective technologies for reducing GHG emissions diminish the costs of low emissions) and $l a$ to be smaller than or equal to $h$ (low emissions cost more than high emissions). Thus, the difference between $h$ and $l a$ represents the minimal costs (e.g., due to missed growth, higher GHG filtering and sequestration costs, taxes, etc.) implied by low emission measures. By contrast, the costs (damages) that can be avoided by keeping the world in a good state are expressed in our stylized decision problem by the difference

$$
1-b
$$

Thus, if $1-b$ is smaller than or equal to $h-l a$, selecting low emissions never pays off. Therefore

$$
b_{c r}=1-(h-l a)
$$

is an important threshold in the parameter space of our emission problem: for values of $b$ between $b_{c r}$ and 1 , selecting low emissions cannot be optimal. In this interval, optimal policies will recommend high GHG emissions. Are there other important thresholds in the problem's parameter space? At this point, we do not know. We have computed optimal policy sequences for a few values of $b$ between 0.5 and 0.91 . These results confirm the analysis and support the conclusion presented above.

The second caveat is that the results presented in Sect. 5.4 offer a rather limited view of the impacts of uncertainties about the implications of exceeding critical thresholds of cumulated GHG emissions on optimal policies. It is true that we have performed more assessments (with probabilities of $5 \%$ and $20 \%$ of turning to a bad world for cumulated emissions below $c r E$; not reported in Sect. 5.4) and that these support the conclusions drawn above.

However, our statistics on the set of possible trajectories associated with a given policy sequence (optimal or not) have been throughout Sect. 5 very rudimentary: we have only assessed the three most likely trajectories, their values and probabilities, and the expected sum of rewards.

In studying the impacts of uncertainties about the implications of exceeding critical thresholds, we have to do with 51200 possible trajectories for every single policy sequence. In this case, more comprehensive statistics would probably be called for. This is computationally challenging; see Section 7.

Thus, the conclusions that we can draw from the results of Sect. 5 are necessarily preliminary. Notice, however, that they are consistent with the analysis reported in Webster (2008) for a two-step decision problem. We are not aware of studies in which the impact of uncertainties on optimal emission policies have been studied systematically for more than two decision steps.

It is probably also fair to point out that, as uncertainties on the implementability of emissions decisions increase and 
(therefore) optimal policies require more and more precautionary approaches, the advantages (in terms of rewards) of earlier emission reductions against delays vanish: in the limit case in which political decisions have no bearing on the measures actually implemented, all policies are optimal.

It should also be kept in mind that in our idealized problem, we have kept the cumulated emission threshold $\operatorname{crE}$ and the critical number of decision steps for technological innovation $c r N$ fixed. In increasing the uncertainty about the availability of technologies for reducing emissions and about the implications of exceeding $\operatorname{crE}$, we have modified the probability distributions below and above $\operatorname{crN}$ and $\operatorname{crE}$ symmetrically. Thus, taking as a reference the certain case, we have increased the probability that efficient technologies will become available before $c r N$ steps from zero to 0.1 and at the same time decreased the probability after $c r N$ from 1 to 0.9 . This is similar for uncertainties on the consequences of exceeding $c r E$. It goes without saying that shifting $c r N$ and $c r E$ does indeed have a strong impact on optimal policies.

Thus, the results presented in Sect. 5 do not imply that improving the accuracy of $c r N$ and $c r E$ estimates is not worth the efforts. But they suggest that obtaining more realistic estimates for the probability of effective technologies for reducing GHG becoming available before and after a critical date is perhaps not as crucial (for computing optimal emission policies for realistic decision problems) as improving our understanding of the implementability of decisions on emission reductions or increases.

Obtaining plausible estimates for the probabilities of being able to implement decisions on emissions reductions or increases naturally brings a political perspective into the problem of computing plausible optimal emission policies.

\section{Future work}

Realistic GHG emission problems involve more than one decision maker (countries) in a competitive situation rather than a single decision maker.

As explained in the Introduction, a generic computational theory for SDPs under uncertainty, multiple players and a finite but unknown number of decision steps is, to the best of our knowledge, still missing. Developing such a theory is a challenging research program. The theory would have to span the border between control and game theory and likely require the introduction of new equilibrium notions. One promising approach towards developing a general theory of optimal decision making is to extend the formalization of SDPs presented in Botta et al. (2017b) using the notions of "quantifier" and "selection function" (together with their respective products) introduced in Escardo and Oliva (2010); Hedges (2017) for infinite horizon open games.

From a more applicational point of view, there are two obvious ways in which the work presented in this paper could be extended to provide more useful insights into the problem of making optimal decisions on emission paths under uncertainty.

One would be to compute optimal emission policies for a realistic emission problem. Besides extending the notions of state and control spaces and, e.g., allowing the decision maker to pick up a few intermediate emission levels between Low and High, this would require assessing the costs and benefits of implementing a given emission level using a realistic integrated assessment model. Such an enterprise would require an interdisciplinary effort on the border between climate science and computing science. Technically, it would require extending the framework for the specification of SDPs SequentialDecisionProblems ${ }^{7}$ with a small domainspecific language for emission problems.

Another way of extending the work presented in this paper would be to keep the focus on stylized emission problems like the one of Sect. 4 but improve the statistical study of the logical consequences of taking decisions according to optimal policy sequences. This could yield tools that support accountable decision making in real-time situations, for instance, during negotiations. Technically, this would imply, among other things, extending SequentialDecisionProblems with algorithms for computing all optimal policies for a given decision problem or perhaps just a certain number of optimal policies.

As we have seen at the end of Sect. 5, computing optimal policies and parsing large collections of possible trajectories or "decision networks" can be computationally challenging even for idealized problems.

Thus, extending SequentialDecisionProblems for computing more optimal policy sequences and more comprehensive statistical analyses of decision networks would benefit from exploiting the concurrency inherent in many of the algorithms presented in Botta et al. (2017b). This is also an interdisciplinary enterprise involving formal methods (concurrent implementations should preserve the machine-checkable optimality proofs that come with the sequential implementation), high-performance computing and climate science.

\footnotetext{
${ }^{7}$ https://gitlab.pik-potsdam.de/botta/IdrisLibs
} 
Appendix A: a summary of Botta et al. (2017a)

The theory presented in Botta et al. (2017a) allows for the specification of SDPs with uncertain outcomes and, for a specific problem, the computation of provably optimal policy sequences and the possible consequences of taking decisions according to an arbitrary policy sequence.

As explained in the Introduction, the theory is dependently typed and the formalization language is Idris; see Brady (2013). Here, we summarize the main requirements and the main results of the theory in a simplified form. For a more detailed discussion of the notion of decision process, decision problem, monadic decision problem, uncertainty, reachability, viability, policy, policy sequence, possible trajectories and avoidability, we refer the reader to Botta et al. (2017a). In a nutshell, a monadic SDP can be specified in terms of the four functions already introduced in Sect. 2:

$$
\begin{aligned}
& \text { State }:(t: \mathbb{N}) \rightarrow \text { Type } \\
& \text { Ctrl }:(t: \mathbb{N}) \rightarrow(x: \text { State } t) \rightarrow \text { Type } \\
& \text { next }:(t: \mathbb{N}) \rightarrow(x: \text { State } t) \rightarrow(y: \text { Ctrl } t x) \rightarrow \\
& M(\text { State }(t+1)) \\
& \text { reward }:(t: \mathbb{N}) \rightarrow(x: \text { State } t) \rightarrow(y: \text { Ctrl } t x) \rightarrow \\
&\left(x^{\prime}: \text { State }(S t)\right) \rightarrow \text { Val }
\end{aligned}
$$

$M$ here is a monad and represents the problem's uncertainties. For deterministic problems (no uncertainties), $M$ is equal to $I d$ and next associates with a state-control pair a unique next state. For nondeterministic problems $M=$ List and for stochastic problems $M=$ Prob. Since $M$ is a monad and therefore a functor, it is equipped with a function

$$
\text { fmap }:(a \rightarrow b) \rightarrow M a \rightarrow M b
$$

that maps functions of type $a \rightarrow b$ for arbitrary $a, b:$ Type to functions of type $M a \rightarrow M b$ and preserves identity and function composition. The type of the values returned by the reward function, $\mathrm{Val}$, is required to be equipped with a zero value zero : Val, with an addition $(\oplus): \mathrm{Val} \rightarrow \mathrm{Val} \rightarrow$ $\mathrm{Val}$ and with a total preorder $(\sqsubseteq)$. Moreover, $\oplus$ is required to be monotonic with respect to $(\sqsubseteq)$ :

$$
\text { monotonePlusLTE : } a \sqsubseteq b \rightarrow c \sqsubseteq d \rightarrow(a \oplus c) \sqsubseteq(b \oplus d)
$$

As mentioned in Sect. 2, a decision maker has also to specify a monotonous measure for weighting uncertain outcomes

$$
\begin{aligned}
\text { meas } \quad: & M \text { Val } \rightarrow \text { Val } \\
\text { measMon }: & \{A: \text { Type }\} \rightarrow \\
& (f: A \rightarrow \text { Val }) \rightarrow(g: A \rightarrow \text { Val }) \rightarrow \\
& ((a: A) \rightarrow(f a) \sqsubseteq(g a)) \rightarrow \\
& (\text { ma }: \text { Prob } A) \rightarrow \\
& \text { meas (fmap } f \text { ma }) \sqsubseteq \text { meas (fmap g ma) }
\end{aligned}
$$

The functions monotonePlusLTE and measMon are examples of specifications: their types formulate properties that $\oplus$, (Б) and meas have to fulfill for the Botta et al. (2017a) theory to be applicable. In this Appendix, we will see further examples of propositional types that encode notions, e.g., of optimality or, as in the case of Bellman, theorems of the Botta et al. (2017a) theory. With the notions of states and controls in place, one can formalize the notions of policy and policy sequence:

$$
\begin{aligned}
& \text { Policy }:(t: \mathbb{N}) \rightarrow \text { Type } \\
& \text { Policy } t=(x: \text { State } t) \rightarrow \text { Ctrl } t x \\
& \text { data PolicySeq }:(t: \mathbb{N}) \rightarrow(n: \mathbb{N}) \rightarrow \text { Type where } \\
& \quad \text { Nil }: \text { PolicySeq } t Z \\
& \quad(:): \text { Policy } t \rightarrow \text { PolicySeq }(S t) m \rightarrow \\
& \quad \text { PolicySeq } t(S m)
\end{aligned}
$$

As discussed in Sect. 2, policies are functions that associate controls with states. They are dependently typed because the domain of Policy $t$ depends on the decision step index $t$. Moreover, its co-domain, $C t r l t x$, depends on $t$ and on $x$.

Policy sequences are just sequences of policies. Since policies are dependently typed functions, we cannot simply collect them in a list or in a vector. The data declaration PolicySeq completely defines the set of all possible policy sequences. In particular, a sequence can only be empty ( $\mathrm{Nil}$, for $n=0$ ) or consist of a head (a policy for taking a decision step at an arbitrary decision step $t$ ) consed (in functional languages, the data constructor (::) is called Cons, and $p:: p s$ is spelled $p$ "consed" with $p s$ ) together with another policy sequence.

For a consistent theory of sequential decision making under uncertainty, the notions of policy and policy sequence actually have to be made more precise. This requires introducing the notions of reachability and viability. In this summary, we omit these important but rather technical aspects, see Botta et al. (2017a). As explained in Sect. 2, the notion of optimality for policy sequences is defined in terms of the measured sum of possible rewards. This is given by a value function

$$
\begin{aligned}
& \text { val }:(x: \text { State } t) \rightarrow \text { PolicySeq } t n \rightarrow \text { Val } \\
& \text { val }\{t\}\{n=Z\} \text { x ps = zero } \\
& \text { val }\{t\}\{n=S m\} x(p:: p s)=\text { meas }\left(\text { fmap } f m x^{\prime}\right) \text { where } \\
& y \quad: \text { Ctrl } t x \\
& y \quad=p x \\
& f \quad: \text { State }(S t) \rightarrow \text { Val } \\
& f x^{\prime}=\text { reward } t x \text { y } x^{\prime} \oplus \text { val } x^{\prime} p s \\
& m x^{\prime}: M(\text { State }(S t)) \\
& m x^{\prime}=\text { next } t x y
\end{aligned}
$$

Notice that when the policy sequence is not empty, the measure meas has to be applied to the result obtained by adding reward t $x$ y $x^{\prime}$ (the reward obtained by selecting the control $y$ in $x$ and for the next state $x^{\prime}$ ) to val $x^{\prime} p s$ (the value of making $m$ decisions according to the policy sequence $p s$ ) for every $x^{\prime}$ in next $t x y$. It is this recursive call of val for every $x^{\prime}$ in next $t x y$ that makes the problem of evaluating policy sequences computationally intractable. For the case in which State $t$ is finite, one can recover linear complexity in $n$ via tabulation. 
The value of policy sequences is the key for formalizing the notion of optimality for policy sequences: a policy sequence for $n$ decision steps is optimal if and only if no other sequence (also for $n$ decisions) attains a higher sum of possible rewards for any state:

$$
\begin{array}{rl}
\text { OptPolicySeq : PolicySeq } t & n \rightarrow \text { Type } \\
\text { OptPolicySeq }\{t\}\{n\} \text { ps }= & \left(p s^{\prime}: \text { PolicySeq } t n\right) \rightarrow \\
& (x: \text { State } t) \rightarrow \\
& \text { val } x s^{\prime} \sqsubseteq \text { val } x p s
\end{array}
$$

The main result of the theory presented in Botta et al. (2017a) is a verified, generic implementation of backwards induction:

$$
\begin{aligned}
& \text { bi }:(t: \mathbb{N}) \rightarrow(n: \mathbb{N}) \rightarrow \text { PolicySeq } t n \\
& \text { bit } Z=\text { Nil } \\
& \text { bit }(S n)=\text { optExt ps }:: \text { ps where } \\
& \quad \text { ps }: \text { PolicySeq }(S t) n \\
& \quad \text { ps }=\text { bi }(S t) n \\
& \text { biLemma }:(t: \mathbb{N}) \rightarrow(n: \mathbb{N}) \rightarrow \text { OptPolicySeq }(\text { bi } t n)
\end{aligned}
$$

The implementation of biLemma relies on the notion of optimal extension of a policy sequence

$$
\begin{aligned}
& \text { OptExt: PolicySeq }(S t) m \rightarrow \text { Policy } t \rightarrow \text { Type } \\
& \begin{aligned}
\text { OptExt }\{t\}\{m\} \text { ps } p= & (x: \text { State } t) \rightarrow \\
& \left(p^{\prime}: \text { Policy } t\right) \rightarrow \\
& \text { val } x\left(p^{\prime}:: p s\right) \sqsubseteq \operatorname{val} x(p:: p s)
\end{aligned}
\end{aligned}
$$

and on a formal proof (that is, a total implementation) of Bellman's principle of optimality (Bellman, 1957):

$$
\begin{aligned}
\text { Bellman : } & (p s: \text { PolicySeq }(S t) m) \rightarrow \text { OptPolicySeq } p s \rightarrow \\
& (p: \text { Policy } t(S m)) \rightarrow \text { OptExt ps } p \rightarrow \\
& \text { OptPolicySeq }(p:: p s)
\end{aligned}
$$

As usual when encoding propositions through types we read Bellman as the first-order logic proposition: for every policy sequence $p s$ and every policy $p$, if $p s$ is optimal and $p$ is an optimal extension of $p s$, then $p:: p s$ is optimal. The implementation of $b i$ relies on optExt: this is a function that takes a policy sequence and computes one of its optimal extensions:

$$
\begin{aligned}
\text { optExt } & : \text { PolicySeq }(S t) n \rightarrow \text { Policy } t(S n) \\
\text { optExtLemma }: & (\text { ps : PolicySeq }(S t) n) \rightarrow \\
& \text { OptExt ps }(\text { optExt ps })
\end{aligned}
$$

Thus, computing the optimal extension of a policy sequence of type PolicySeq $(S t) n$ implies solving an optimization problem for every state in State $t$. If the set of control Ctrl $t x$ is finite for a given $x$ : State $t$, this problem can be solved by linear search. A further result of the theory presented in Botta et al. (2017a) is a generic algorithm for computing all possible trajectories that can be obtained by applying a policy sequence (optimal or not) starting from a given state or, if the decision maker takes decisions with imperfect information, from an $M$ structure of states. In both cases, the result is an $M$ structure of state-control sequences:

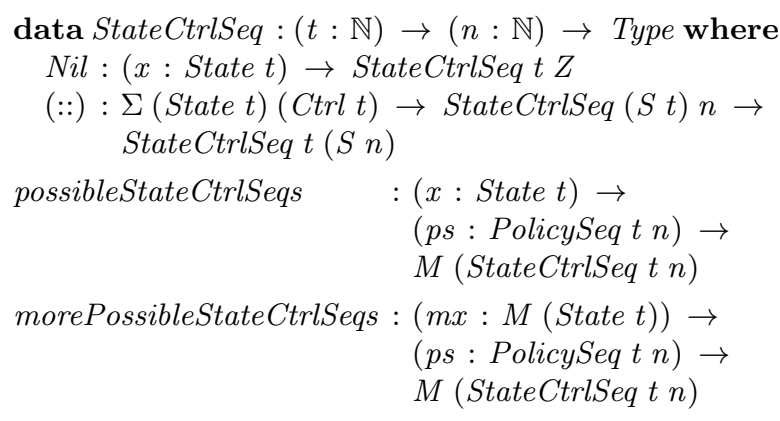

For the implementations of biLemma, Bellman, possibleStateCtrlSeqs and morePossibleStateCtrlSeqs we refer the reader to Botta et al. (2017a). In Sect. 5 we make extensive usage of, among other things, $b i$ and possibleStateCtrlSeqs for computing optimal emission policies and possible state-control sequences. 
Competing interests. The authors declare that they have no conflict of interest.

Acknowledgements. The authors thank the ESD editors and reviewers, whose comments have lead to significant improvements of the original manuscript. The work presented in this paper heavily relies on free software, among others on Idris, Agda, GHC, git, vi, Emacs, $\mathrm{AT}_{\mathrm{E}} \mathrm{X}$ and on the FreeBSD and Debian GNU/Linux operating systems. It is our pleasure to thank all developers of these excellent products. This work was partially supported by the projects GRACeFUL (grant agreement No 640954) and CoeGSS (grant agreement No 676547), which have received funding from the European Union's Horizon 2020 research and innovation programme.

The article processing charges for this open-access publication were covered by the Potsdam Institute for Climate Impact Research (PIK).

Edited by: Michel Crucifix

Reviewed by: Michel Crucifix and one anonymous referee

\section{References}

Bellman, R.: Dynamic Programming, Princeton University Press, Princeton, 1957.

Botta, N.: IdrisLibs, v1.2.0, https://gitlab.pik-potsdam.de/botta/ IdrisLibs 2018-05-14, 2016-2018.

Botta, N., Jansson, P., and Ionescu, C.: Contributions to a computational theory of policy advice and avoidability, J. Funct. Program., 27, Oct. 2017, https://doi.org/10.1017/S0956796817000156, 2017a.

Botta, N., Jansson, P., Ionescu, C., Christiansen, D. R., and Brady, E.: Sequential decision problems, dependent types and generic solutions, Log. Meth. Comput. Sci., 13 (1), https://doi.org/10.23638/LMCS-13(1:7)2017, 2017b.

Brady, E.: Programming in Idris: a tutorial, http://docs.idris-lang. org/en/latest/tutorial/index.html\#tutorial-index 2018-05-14, 2017.

Brady, E.: Type-Driven Development with Idris, Manning Publications, Shelter Island, 2017.

Curry, H. B.: Outlines of a Formalist Philosophy of Mathematics, 2nd Edn., North-Holland Publishing Company, Amsterdam, 1958.

Escardo, M. and Oliva, P.: Selection functions, bar recursion and backward induction, Math. Struct. Comput. Sci., 20, 127-168, https://doi.org/10.1145/1167473.1167499, 2010.

Finus, M., van Ierland, E., and Dellink, R.: Stability of Climate Coalitions in a Cartel Formation Game, FEEM Working Paper No. 61.2003, http://ssrn.com/abstract=447461 2018-05-14, 2003.

Fudenberg, D. and Tirole, J.: Game Theory, MIT Press, Cambridge Massachusetts, 1991.

Hardin, G.: The Tragedy of the Commons, Science, 162, 12431248, 1968.

Hedges, J.: Towards compositional game theory, $\mathrm{PhD}$ thesis, https: //qmro.qmul.ac.uk/xmlui/handle/123456789/23259 2018-05-14, 2017.
Heitzig, J., Lessmann, K., and Zou, Y.: Self-enforcing strategies to deter free-riding in the climate change mitigation game and other repeated public good games, PNAS, 108 (38), 15739-15744, https://doi.org/10.1073/pnas.1106265108, 2011.

Helm, C.: International emissions trading with endogenous allowance choices, J. Publ. Econ., 87, 2737-2747, 2003.

Ionescu, C.: Vulnerability Modelling and Monadic Dynamical Systems, PhD thesis, Freie Universität Berlin, Berlin, https://d-nb. info/1023491036/34 2018-05-14, 2009.

Ionescu, C. and Jansson, P.: Testing versus proving in climate impact research, in: Proc. TYPES 2011, vol. 19 of Leibniz International Proceedings in Informatics (LIPIcs), Schloss Dagstuhl - Leibniz-Zentrum fuer Informatik, Dagstuhl, Germany, 41-54, https://doi.org/10.4230/LIPIcs.TYPES.2011.41, 2013.

Norell, U.: Towards a practical programming language based on dependent type theory, PhD thesis, Chalmers University of Technology, https://doi.org/10.1.1.65.7934, 2007.

Intergovernmental Panel on Climate Change: Climate Change 2007: Synthesis Report, in: Contributions of Working Groups I, II and III to the Fourth Assessment Report of the Intergovernmental Panel on Climate Change, Cambridge University Press, Cambridge, United Kingdom and New York, NY, USA, 2007.

Parson, E. A. and Karwat, D.: Sequential Climate Change Policy, Wiley Interdisciplin. Rev.: Clim. Change, 2, 744-56, https://doi.org/10.1007/s10666-005-9014-6, 2011.

Schönfinkel, M.: Über die Bausteine der mathematischen Logik, Mathematische Annalen, 92/93, 305-316, 1924.

Peterson, S.: Uncertainty and economic analysis of climate change: A survey of approaches and findings, Environ. Model. Assess., 11 (1), 1-17, 2006.

Webster, M. D.: The Curious Role of "Learning" in Climate Policy: Should We Wait for More Data?, The Energy Journal, 23 (2), 97-119, www.jstor.org/stable/41322956, 2002.

Webster, M. D.: Incorporating Path Dependency into DecisionAnalytic Methods: An Application to Global Climate-Change Policy, Decis. Anal., 5, 60-75, 2008. 